\title{
Research of the process of radon accumulation in the accumulating chamber taking into account the nonlinearity of its entrance
}

\author{
Dmitriy Tverdyi ${ }^{1, \star}$, Roman Parovik ${ }^{2, \star \star}$, Evgeniy Makarov ${ }^{3, \star \star \star}$ Pavel Firstov ${ }^{3, \star \star \star \star}$ \\ ${ }^{1}$ Vitus Bering Kamchatka State University, 683032, Petropavlovsk-Kamchatsky, Pogranichnaya str. 4, \\ Russia \\ ${ }^{2}$ Institute of Cosmophysical Research and Radio Wave Propagation of the Far Eastern Branch of Russian \\ Academy of Science, 684034, Kamchatskiy kray, Paratunka, Mirnaya str. 7, Russia. \\ ${ }^{3}$ Kamchatka Branch, Geophysical Survey, Russian Academy of Sciences, 683006, Petropavlovsk- \\ Kamchatsky, 9 Piip Boulevard, Russia.
}

\begin{abstract}
The paper presents a mathematical model of radon accumulation in a chamber, which takes into account the hereditary properties of the environment in which radon migrates, and also uses a nonlinear function that is responsible for the mechanisms of radon entering the chamber. The simulation of accumulation is performed in comparison with real data. It is shown that the model presented in this work gives a better agreement between the model and real curves of radon accumulation and can be used for a more accurate description of the processes occurring in the chamber.
\end{abstract}

\section{Introduction}

Monitoring of the radon field in the upper soil layer is of interest both in connection with the effect of variations in meteorological quantities on the radon runoff into the atmosphere, and with the influence on its dynamics of changes in the stress-strain state of the geoenvironment in order to predict strong earthquakes. Variations of the regional stress field that precede earthquakes find a response in the upper soil layer, which leads to changes in porosity, temperature and pressure gradients, and, consequently, to a change in the rate of radon migration to the day surface. Therefore, the monitoring of the parameters of the radon field at the boundary of the "lithosphere - atmosphere" allows us to talk about the processes occurring in the earth's crust, which can cause anomalous variations in the concentration of radon, as well as changes in the magnitude of the radon flux density (RFD) from the soil surface $[1,3]$.

At the Petropavlovsk-Kamchatskiy geodynamic test site, since 1997, the registration of subsoil $\mathrm{Rn}$ has been carried out in order to search for precursor anomalies of strong earthquakes [2].

In this paper, using mathematical modeling, the process of radon accumulation in a storage chamber is considered, where gas-discharge meters are installed in order to monitor subsoil radon. The

\footnotetext{
^e-mail: dimsolid95@mail.ru

$\star \star$ e-mail: romanparovik@gmail.com

$\star \star \star$ e-mail: ice@emsd.ru

$\star \star \star \star$ e-mail: firstov@emsd.ru
} 
concentration of radon inside the chambers installed on the ground is the result of two main competing processes: the influx of radon and its runoff. Radon intake is due to the presence of: sources of radon intake, pathways of entry, and a driving force that induces radon to enter the chamber. Radon migrates from the soil under the chamber through pores, cracks and air cavities. The processes occurring in this case are due to the presence of a gradient of radon concentration in the medium and a pressure gradient. In the first case, the process of diffusion transfer is formed, in the second convective (gas transfer in a gas) or filtration (gas transfer in a porous medium).

When organizing monitoring points for subsoil radon in order to expand the network, it is necessary to know the value RFD from the surface at the selected point where the storage chamber will be installed. RFD on a qualitative level, it determines the tensosensitivity of the selected point, which depends on the convective rate of radon transfer and the magnitude of its volumetric activity. The zones of dynamic influence of the fault are characterized by areas of decompaction with increased flow Rn, which respond well to changes in the stress-strain state of the geomedium; they are most suitable for registration of radon. In addition, the areas of subsoil radon runoff into the atmosphere are characterized by narrowly localized zones, the search for which determines the need for a quick assessment of the RFD by a simple and accessible method. This method, based on the mathematical description of the accumulation of $\mathrm{Rn}$ in the chamber, according to the approach from [4], was described in detail in [2]. This method was tested at the points of the subsoil gas monitoring network in Kamchatka [2].

This approach requires that the chamber there was a hole allowing to ensure its ventilation. There is no air exchange in a closed chamber, therefore, when setting a boundary value problem, there is no parameter of the air exchange rate (AER). The accumulation of soil radon in a closed chamber is due to Fick's law - in a state of radon saturation, due to the absence of a gradient of the OA Rn difference at the "soil-chamber" boundary, the flow of radon from the soil into the chamber stops. After installing the camera on the ground, the time to reach the saturation state is determined by the height of the camera and the diffusion coefficient of radon in the ground, as well as by the convection rate, if this component of the transfer is important (the RFD value does not matter). Thus, long-term monitoring of radon in a closed chamber (without a hole, in a state of radon saturation) is analogous to monitoring $\mathrm{Rn}$ in the soil at some shallow depth, and is not monitoring the radon flux density from the soil surface. Periodic ventilation of the chamber and measurement of the dynamics of radon concentration only in the initial periods of accumulation makes it possible to control the dynamics of RFD, but this is a methodically complex approach.

In the mathematical description of the process of radon accumulation in the chamber in [2], a number of assumptions were used to simplify the solution of the problem, and the accumulation process itself was assumed to be stationary, when there are no dramatically changing conditions of AER and RFD from the surface below it. Consequently, the possible factors affecting the flow rate were not taken into account, primarily variations in atmospheric pressure and temperature. In the chamber used in the work, there is air exchange with atmospheric air present but minimized. Due to weather conditions (mainly due to temperature fluctuations), the AER will vary significantly.

For a more accurate description of the accumulation process, the existing model was developed using a nonlinear function responsible for the mechanisms of Rn entering the chamber, as well as taking into account heredity.

\section{Mathematical model}

Mathematical modeling of the transfer of $\mathrm{Rn}$ in porous soil due to the mechanisms of diffusion and convection, taking into account the hereditary properties of the medium, was considered in [5, 6]. Here, the hereditary properties of the porous medium were due to the presence of isolated pores and 
conducting channels between them. In the first case, Rn can be retained in isolated pores for a long time, and in the second case, it can quickly migrate through space (Levy flights). Such mechanisms of $\mathrm{Rn}$ transfer in the literature are called subdiffusion and superdiffusion or anomalous diffusion [7]. It should be noted that the mathematical description of anomalous diffusion is given using derivatives of fractional orders, which are investigated in the framework of fractional calculus [8]. In this work, we will consider a slightly different hereditary process of radon migration in a chamber.

Consider the following law of accumulation radon volumetric activity (RVA) in the chamber:

$$
\frac{d A}{d t}=\int_{0}^{t} K(t-\tau) F(A, \tau) d \tau, A(0)=A_{0}
$$

where $A(t)$ - RVA; $F(A, t)$ - the function responsible for the mechanisms of Rn entering the chamber and in the general case can be nonlinear; $K(t-\tau)$ - memory function, determines the degree of influence of the function $F(A, t)$ on the accumulation process; $A_{0}-$ at the initial moment of time $t$.

Note that there can be two limiting cases when $K(t-\tau)=\delta(t-\tau), \delta(\cdot)$ - the Dirac function and $K(t-\tau)=t-H(t-\tau), H(\cdot)-$ Heaviside function. In the first case, the memory effect, by analogy with Markov processes, is absent, and in the second case it characterizes the presence of ideal memory. In general, the memory function $K(t-\tau)$ in equation (1) can be selected based on experimental data or properties of the medium.

Due to the fact that the rate of radon intake decreases with time, the faster, the smaller the AER of the chamber with the atmosphere, then we will consider the dynamic system (1) dissipative. Here, by dissipation, we mean that the system gradually "forgets" the mechanisms of radon entry into the chamber, i.e. RVA goes over time to a steady state (saturation). In the first approximation, for the dissipative system (1), the memory function $K(t-\tau)$ can choose as:

$$
K(t-\tau)=\frac{1}{\Gamma(\alpha)(t-\tau)^{1-\alpha}}, 0<\alpha \leq 1,
$$

where $\alpha$ - determines the intensity of dissipation, $\Gamma(\cdot)$ - gamma function.

Substituting relation (2) into equation (1), we obtain

$$
\frac{d A}{d t}=I_{0 t}^{\alpha} F(A, \tau), A(0)=A_{0},
$$

where $I_{0 t}^{\alpha} F(A, \tau)=\frac{1}{\Gamma(\alpha)} \int_{0}^{t} \frac{F(A, \tau)}{(t-\tau)^{1-\alpha}} d \tau-$ fractional integral of order $\alpha$. If we invert equation (3) taking into account the composition property of the fractional integral [8], we get the following equation:

$$
\partial_{0 t}^{\alpha} A(\tau)=F(A, t), A(0)=A_{0}
$$

where $\partial_{0 t}^{\alpha} A(\tau)=\frac{1}{\Gamma(1-\alpha)} \int_{0}^{t} \frac{\dot{A}(\tau) d \tau}{(t-\tau)^{\alpha}}$ - fractional derivative of order $\alpha, \dot{A}(\tau)=d A / d t$.

Now, if in (4) choose the function $F(A, t)=S-\lambda_{0} A(t)$, where $S$ - diffusion mechanism of RVA entry, $\lambda_{0}-\mathrm{AER}$, we get

$$
\partial_{0 t}^{\alpha} A(\tau)=S-\lambda_{0} A(t), A(0)=A_{0} .
$$

The solution to problem (5) is given in terms of the special Mittag-Leffler function [8]:

$$
A(t)=A_{0} E_{\alpha}\left(-\lambda_{0} t^{\alpha}\right)+A_{\max } t^{1-\alpha}\left(1-E_{\alpha}\left(-\lambda_{0} t^{\alpha}\right)\right),
$$


where $E_{\alpha}\left(-\lambda_{0} t^{\alpha}\right)=\sum_{k=0}^{\infty} \frac{\left(-\lambda_{0} t^{\alpha}\right)^{k}}{\Gamma(\alpha k+1)}-$ Mittag-Leffler function, $A_{\max }=S / \lambda_{0}$.

Note that in the case when in solution (6) $\alpha=1$, we obtain the known solution [2]:

$$
A(t)=A_{0} \exp \left(-\lambda_{0} t^{\alpha}\right)+A_{\max }\left(1-\exp \left(-\lambda_{0} t^{\alpha}\right)\right)
$$

\section{Simulation results}

The data on the accumulation of radon in the chambers at the PRTR point (Kamchatka) and the YSSR point on Sakhalin Island were used as the initial data for modeling [9]. In these points continuously record radon in chambers at depths from 1 to $3 \mathrm{~m}$ [2]. The chambers are a 10 liter bucket. A radon sensor is placed in this chamber through a special pipe. The minimum air exchange between the chamber and the atmosphere is also carried out through the pipe. The curves of radon accumulation in the chambers at the PRTR and YSSR stations and the simulation results are shown in Figure 1 and Figure 2.

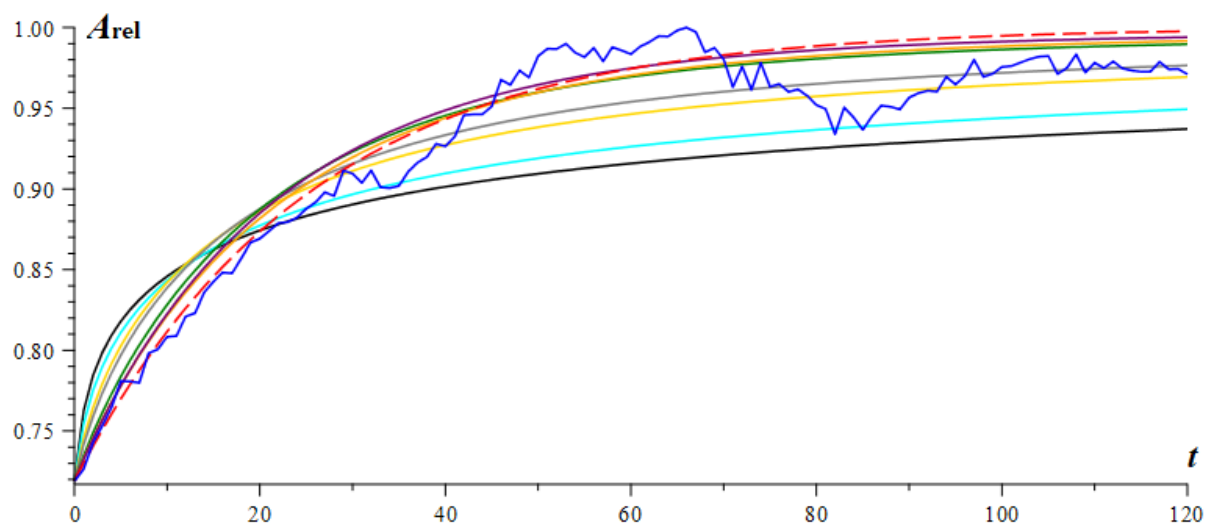

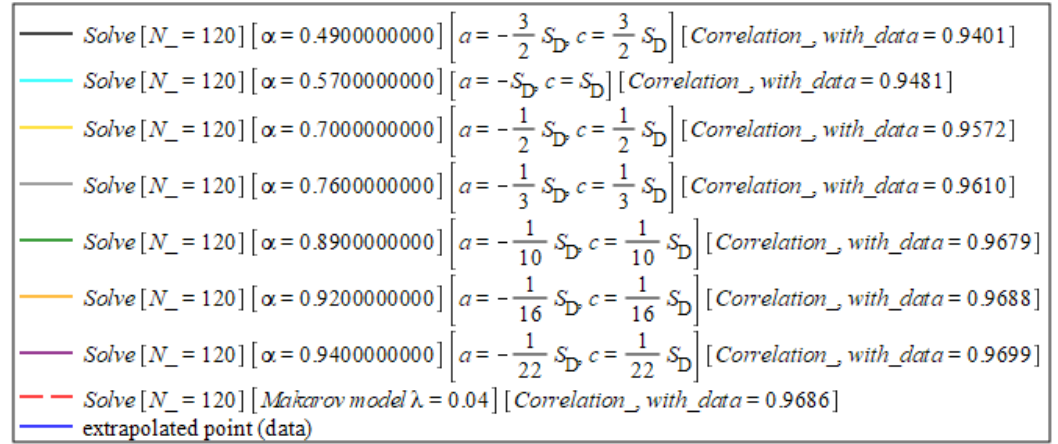

Figure 1. Radon accumulation data in the chamber at the PRTR point (navy blue curve) and model curves with different parameter $\alpha$.

Figure 1 and Figure 2 it can be seen that the calculated curves obtained by the model (5) at various values of the parameter $\alpha$ are rearranged in the reverse order, and their tails are tightened. This regularity characterizes the memory effect and determines the power law according to (6). In Figure 1 


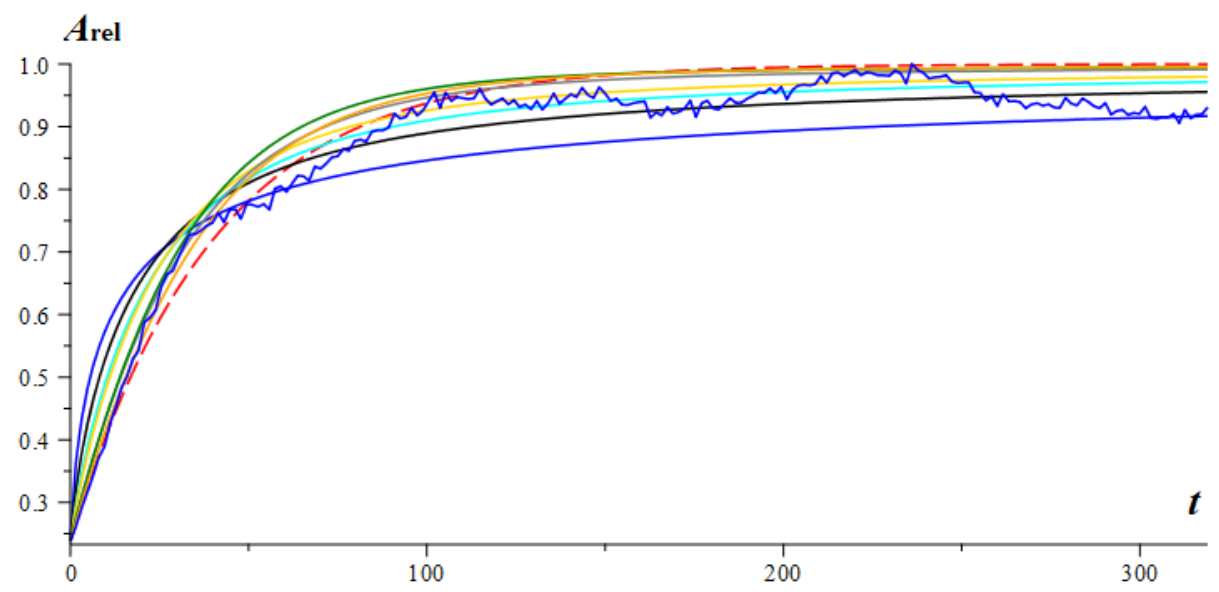

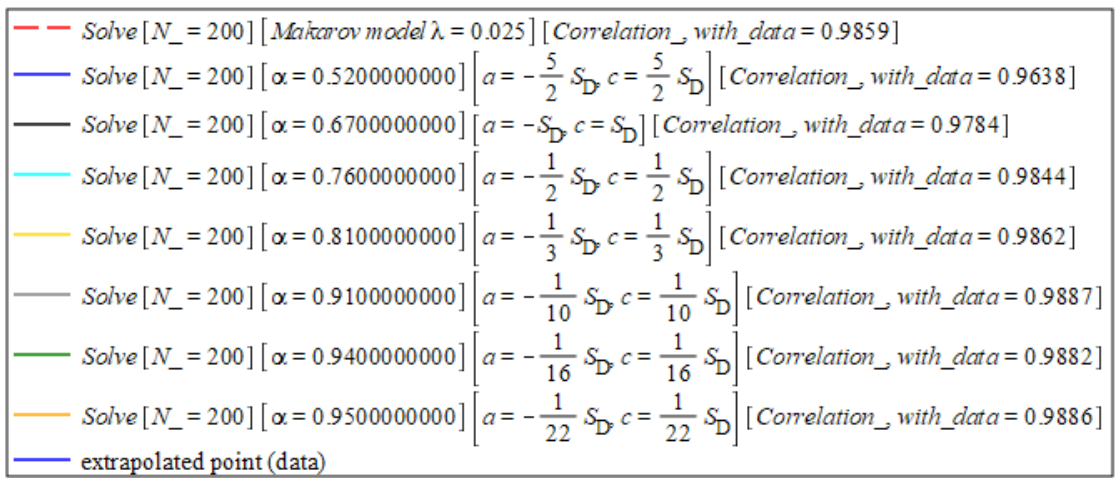

Figure 2. Radon accumulation data in the chamber at the YSSR point (navy blue curve) and model curves with different parameter $\alpha$.

and Figure 2, we see that the best approximation to the experimental data, in contrast to the classical model $(\alpha=1)$ give calculated curves with parameters $\alpha=0.94$ and $\alpha=0.95$. Therefore, we can conclude that the introduction of the effect of heredity into the equation gives an additional degree of freedom, which means that it allows more flexible modeling of the process of radon accumulation in the chamber.

\section{Conclusion}

The paper proposes a new mathematical model for the accumulation of RVA taking into account heredity. It is shown that the proposed model can, due to an additional parameter - the intensity of dissipation of the accumulation mechanism, give results that are closer to real data, in comparison with the classical model from [2]. 


\section{Acknowledgement}

This work was partially supported by the Russian Foundation for Basic Research (grant no. 20-0500493).

\section{References}

[1] Firstov P.P., Makarov E.O., Seismic instruments, 51, 58-80 (2015)

[2] Firstov P.P., Makarov E.O., Subsoil radon dynamics in Kamchatka and strong earthquakes (KamSU named after Vitus Bering, Petropavlovsk-Kamchatsky, 2018), 148

[3] Cicerone R.D., Ebel J.E., Beitton J., Tectonophysics, 476, 371-396 (2009)

[4] Vasilyev A.V., Zhukovsky M.V., Journ. Environmental Radioactivity, 124, 185-190 (2013)

[5] Parovik R.I., Shevtsov B.M, Mathematical Models and Computer Simulations, 2, 180-185 (2010)

[6] Parovik R.I., Life Science Journal, 11, 281-283 (2014)

[7] Uchaikin V.V., Fractional derivatives for physicists and engineers. Volume I. (Springer, Berlin, 2013), 355

[8] Kilbas A.A., Srivastava H.M., Trujillo J.J., Theory and Applications of Fractional Differential Equations (Elsevier, Amsterdam, 2006), 523

[9] Makarov E., Firstov P., Kostylev D., Rylov E., Dudchenko I., E3S Web of Conferences, 62, 03007, 2018 